\title{
Noncollinear magnetism in nanosized cobalt chromite
}

\author{
D. Zákutná, ${ }^{1,2, *}$ J. Vlček, ${ }^{3}$ P. Fitl,${ }^{3}$ K. Nemkovski, ${ }^{4}$ D. Honecker, ${ }^{5}$ D. Nižňanský, ${ }^{1,6}$ and S. Disch ${ }^{2, \dagger}$ \\ ${ }^{1}$ Department of Inorganic Chemistry, Faculty of Science, Charles University in Prague, Hlavova 2030/8, 12843 Prague 2, Czech Republic \\ ${ }^{2}$ Department für Chemie, Universität zu Köln, Luxemburger Strasse 116, 50939 Köln, Germany \\ ${ }^{3}$ Department of Physics and Measurements, University of Chemistry and Technology Prague, Technicka 5, 16628 Prague, Czech Republic \\ ${ }^{4}$ Forschungszentrum Jülich GmbH, Jülich Centre for Neutron Science (JCNS) at Heinz Maier-Leibnitz. \\ Zentrum (MLZ), Lichtenbergstr. 1, 85748 Garching, Germany \\ ${ }^{5}$ Institut Laue-Langevin, 71 Avenue des Martyrs, F-38042 Grenoble, France \\ ${ }^{6}$ Institut of Inorganic Chemistry of the AS CR, v.v.i., 25068 Husinec-Rež, Czech Republic
}

(Received 3 May 2018; revised manuscript received 10 June 2018; published 6 August 2018)

\begin{abstract}
Using a combination of neutron diffraction with XYZ polarization analysis and magnetization measurements, the noncollinear magnetism in nanosized cobalt chromite, a potential multiferroic material, is revealed. For noninteracting 26.9(1) $\mathrm{nm}$ nanoparticles, a bulklike behavior is identified, including a ferrimagnetic Curie temperature of $99 \mathrm{~K}$ and a transition to the spin spiral magnetic phase at $27 \mathrm{~K}$ with a temperature-dependent, incommensurate propagation vector. A lock-in transition towards a commensurate propagation vector is not observed. Much smaller, 3.1(2) nm, nanoparticles reveal a strong cluster glass behavior, characterized by ferrimagnetic behavior below the Curie temperature of $43 \mathrm{~K}$ and a transition to asperomagnetic behavior at $18 \mathrm{~K}$, with the absence of any magnetic reflections at a base temperature of $5 \mathrm{~K}$.
\end{abstract}

DOI: 10.1103/PhysRevB.98.064407

\section{INTRODUCTION}

Multiferroic materials with coupling of two or more ferroic properties attract great interest in terms of both fundamental physics research and potential applications, e.g., spintronics $[1,2]$. Many multiferroics, e.g., $\mathrm{BiFeO}_{3}$, are naturally ferroelectric with magnetic ions, with a subclass of coupled materials such as $\mathrm{RMnO}_{3}$ (where $\mathrm{R}=\mathrm{Tb}$, Dy, and Ho), $\mathrm{Ni}_{3} \mathrm{~V}_{2} \mathrm{O}_{8}$, or $\mathrm{TbMn}_{2} \mathrm{O}_{5}$, which exhibit electric polarization induced by magnetically ordered states, but without spontaneous magnetization [3-7]. Multiferroic systems that exhibit strong magnetoelectric coupling include spiral magnets, in which ferroelectricity is induced by spin spiral magnetic order. The onset of ferroelectric polarization appears with the transition to a spiral magnetic phase due to strong geometrical frustration or distortion [8]. A material with both spontaneous magnetization in combination with electric polarization of spin spiral origin is cobalt chromite $\left(\mathrm{CoCr}_{2} \mathrm{O}_{4}\right)$ [9-12]. In addition, a dielectric anomaly below the spiral magnetic phase has been observed in polycrystalline samples [13] as well as in single crystals $[9,10,14]$. The ferroelectricity in $\mathrm{CoCr}_{2} \mathrm{O}_{4}$ is affected by a spin spiral magnetic phase evolved by strong near-neighbor exchange interactions and magnetic geometrical frustration on the spinel B site. Few reports exist on magnetic properties and phases in nanoparticles (NPs) of $\mathrm{CoCr}_{2} \mathrm{O}_{4}$ [15-18]. A magnetic neutron diffraction study of the magnetic phase transitions in nanosized $\mathrm{CoCr}_{2} \mathrm{O}_{4}$ was done for $\mathrm{CoCr}_{2} \mathrm{O}_{4}$ mixed with

*Present address: Institut Laue-Langevin, F-38042 Grenoble, France and Department für Chemie, Universität zu Köln, Luxemburger Strasse 116, 50939 Köln, Germany.

${ }^{\dagger}$ Corresponding author: sabrina.disch@uni-koeln.de
0.1 of $\mathrm{Fe}$ [19] on octahedral site, as well as pure $\mathrm{CoCr}_{2} \mathrm{O}_{4}$ NPs with substantial aggregate formation [20,21] revealing bulklike behavior along with diffuse scattering for $10 \mathrm{~nm}$ NPs. In the present work we report on the magnetic phase transitions observed in cobalt chromite NPs with crystallite sizes in the intermediate range between the amorphous disordered state and polycrystalline regime, by means of polarized neutron diffraction and macroscopic magnetization measurements. Emphasis is on the in-depth characterization of the spin-spiral ordering and the evolution of the propagation vector by using a conical spin model.

\section{EXPERIMENT}

Two samples of cobalt chromite NPs were synthesized by the nonalkoxide sol-gel method [22] with an epoxide as gelation agent as well as using the high temperature hydrothermal method [18]. In case of the sol-gel method, $0.87 \mathrm{~g}$ ( $3 \mathrm{mmol})$ of $\mathrm{Co}\left(\mathrm{NO}_{3}\right)_{2}$ and $2.08 \mathrm{~g}(6 \mathrm{mmol})$ of $\mathrm{Cr}\left(\mathrm{NO}_{3}\right)_{3}$ were dissolved in $15 \mathrm{~mL}$ of ethanol, and $4.96 \mathrm{~g}$ of the gelation agent (propylene oxide) was added. The dark green gel formed after $21 \mathrm{~min}$ was aged in a closed vessel at $50{ }^{\circ} \mathrm{C}$ for $2 \mathrm{~h}$ and dried in an open vessel at $50{ }^{\circ} \mathrm{C}$ for $24 \mathrm{~h}$. After subsequent annealing of the dried black-green gel at $600{ }^{\circ} \mathrm{C}$ for $5 \mathrm{~h}$, a dark green powder of crystalline cobalt chromite NPs was obtained (sample S1). In high temperature hydrothermal synthesis, cobalt(II) and chromium(III) chloride precursors (0.95 g, $2 \mathrm{mmol}$, of $\mathrm{CoCl}_{2} \cdot 6 \mathrm{H}_{2} \mathrm{O}$ and $1.066 \mathrm{~g}, 4 \mathrm{mmol}$, of $\mathrm{CrCl}_{3}$. $6 \mathrm{H}_{2} \mathrm{O}$ ) were used in order to prepare the corresponding metal oleate complexes. Oleates were decomposed in an autoclave in a water/ethanol mixture (1:2) at high pressure (47 bars) and temperature $\left(247{ }^{\circ} \mathrm{C}\right)$. The resulting NPs were washed three times by dispersing in hexane and precipitating with ethanol. 
A powder of cobalt chromite NPs was prepared by evaporation of the obtained blue-green dispersion (sample S2).

Transmission electron microscopy (TEM) was carried out using a ZEISS LEO 902 operated at $120 \mathrm{kV}$ with $\mathrm{LaB}_{6}$ cathode in a bright field (BF) mode. The sample was deposited onto a coated copper grid. The mean particle diameter was statistically determined from at least 200 particles in different $\mathrm{BF}$ images and fitted with a log-normal distribution function. Only isolated particles without overlap were counted in order to obtain precise size determination.

Powder x-ray diffraction (PXRD) was measured using a PANalytical X'Pert PRO diffractometer with $\mathrm{Cu} K_{\alpha}$ radiation $(\lambda=1.54 \AA)$ equipped with a secondary monochromator and a PIXcel detector. The sample was measured in the $Q$ range of $1-5 \AA^{-1}$ with a step size of $0.003 \AA^{-1}$. The particle diameter was determined by Rietveld analysis, using the FullProf software [23]. The spinel structure was refined by Rietveld analysis using a pseudo-Voigt profile function. The instrumental broadening was determined using a $\mathrm{LaB}_{6}$ reference (SR 660b, NIST).

Macroscopic magnetization measurements were carried out using a Quantum Design Physical Property Measurement System (PPMS) equipped with a superconducting magnet and a vibrating sample magnetometer (VSM) option. The zero-field-cooled (ZFC) and field-cooled (FC) magnetization measurements were done between 2 and $300 \mathrm{~K}$ with a heating rate of $1 \mathrm{~K} / \mathrm{min}$ in an applied magnetic field of $\mu_{0} H=10 \mathrm{mT}$. Isothermal, field dependent magnetization measurements were carried out at 2, 10, 50, and $100 \mathrm{~K}$. In the superparamagnetic regime, a Langevin fit was performed according to

$$
L(\xi)=\operatorname{coth}(\xi)-1 / \xi
$$

with $\xi=\frac{\mu \mu_{0} H}{k_{\mathrm{B}} T}$, where $\mu$ is the particle magnetic moment, $\mu_{0}$ is the permeability of vacuum, $k_{\mathrm{B}}$ is the Boltzmann constant, and $T$ is the temperature. A particle dispersion of the $\mathrm{S} 2$ sample was measured in a closed cycle measurement system (CCMS) by Cryogenics Ltd. using a vespel ${ }^{\circledR}$ sample holder. Temperature-dependent ac susceptometry was measured on a Quantum Design MPMS 7XL device (SQUID) in zero external magnetic field at frequencies of $v=0.1,1,10,100$, and $893 \mathrm{~Hz}$ with an ac magnetic amplitude of $0.1 \mathrm{mT}$.

Polarized neutron scattering with XYZ polarization analysis was carried out at the diffuse neutron scattering (DNS) instrument $[24,25]$ at Heinz Maier-Leibnitz Zentrum (MLZ). The six axial polarization-resolved scattering contributions were collected for $\mathrm{S} 1$ and $\mathrm{S} 2$ samples as well as a $\mathrm{NiCr}$ standard and vanadium reference over a scattering momentum range of $0.5<Q<3.4 \AA^{-1}$. The reference measurement of $\mathrm{NiCr}$ alloy yields the flipping ratio correction and thus the real polarization of the incident neutron beam. Vanadium was measured to calibrate the detector efficiency. The measurements were carried out using aluminum sample holders in a temperature range of $5-100 \mathrm{~K}$ using a neutron wavelength of $\lambda=3.3 \AA$. The magnetic, nuclear coherent and nuclear spin-incoherent contributions were separated from the total scattering cross section by means of XYZ-polarization analysis [26]. Separation of the observed data was performed using polarization analysis algorithm, implemented in the software dnsplot [27]. LeBail fits of the magnetic and nuclear coherent scattering
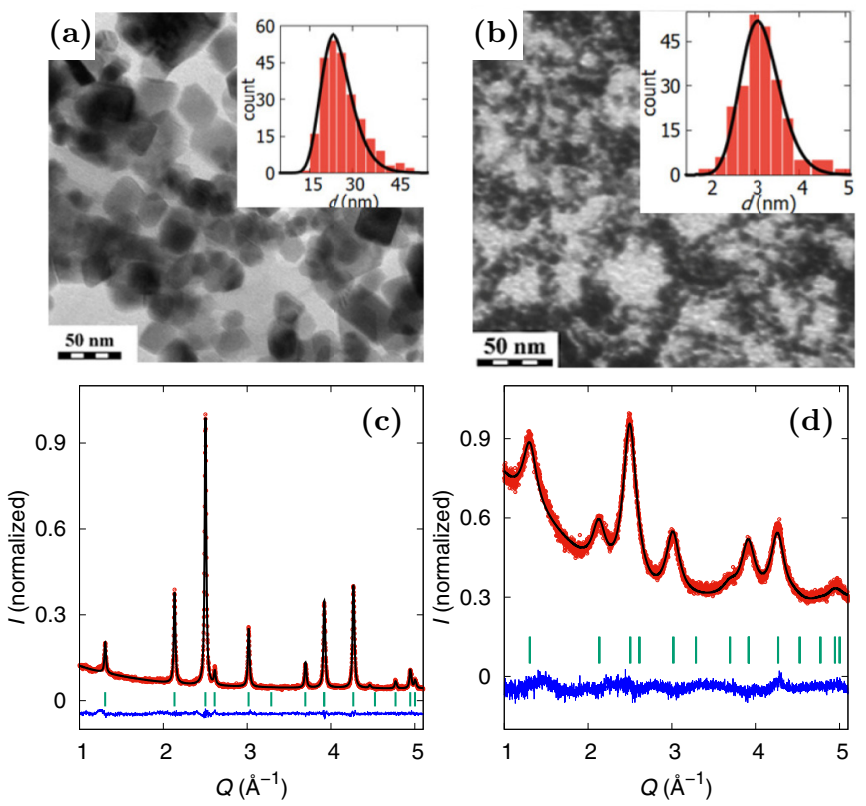

FIG. 1. Bright field (BF) TEM micrographs of (a) S1 and (b) S2 samples with the lognormal size distribution as insets. (c) and (d) Rietveld analysis of prepared $\mathrm{CoCr}_{2} \mathrm{O}_{4}$ NPs. The red points correspond to experimental data, black line is Rietveld fit, the blue line represents the residual, and green vertical lines correspond to the Bragg peak positions of spinel $\mathrm{CoCr}_{2} \mathrm{O}_{4}$ structure.

cross sections were done using the Thompson-Cox-Hastings pseudo-Voigt profile function [28].

\section{RESULTS AND DISCUSSION}

Cobalt chromite NPs were synthesized with two different particle sizes according to the synthesis routes. Using the alkoxide sol-gel method with a high temperature annealing step, nearly spherical NPs with a mean particle diameter of $d_{\mathrm{S} 1}=26.9(1) \mathrm{nm}$ are obtained [S1 sample, Fig. 1(a)]. The large lognormal particle size distribution of $\sigma_{\log }=0.23$ is likely a result of the sintering process. X-ray powder diffraction with Rietveld analysis [Fig. 1(c), Table I] confirms a normal $\mathrm{CoCr}_{2} \mathrm{O}_{4}$ spinel structure $\left(\mathrm{AB}_{2} \mathrm{X}_{4}\right)$ within the cubic $F d \overline{3} m$ space group (227), where Co occupies the $\mathrm{A}$ sites and $\mathrm{Cr}$ the B sites. The determined coherent grain size of 21.9(4) $\mathrm{nm}$ is slightly smaller than the particle diameter from TEM, indicating surface disorder. Significantly smaller NPs were synthesized using a high temperature hydrothermal technique [S2 sample, Figs. 1(b) and 1(d)]. A mean particle diameter of $d_{\mathrm{S} 2}=3.1(2) \mathrm{nm}$ with a smaller lognormal size distribution of $\sigma_{\log }=0.12$ is obtained. The structurally coherent grain size of 2.9(1) $\mathrm{nm}$ is in excellent agreement with the morphology determined using TEM. From Rietveld analysis, a lattice parameter of $a=8.351$ (1) $\AA$ was obtained, which is larger as compared to the S1 sample with $a=8.333(3) \AA$, that is in good agreement with bulk value of $a=8.33 \AA$ [11]. This suggests a significant degree of structural disorder in the S2 sample which may be related to the considerably lower synthesis temperature. These two samples of $\mathrm{CoCr}_{2} \mathrm{O}_{4}$ with clearly different particle sizes in the intermediate range 
TABLE I. Refined parameters from Rietveld analysis. The Lorentzian broadening is given by particle size broadening $(Y)$. The parameter BOV represents an overall isotropic displacement, $u$ is the oxygen site, and $C o_{\mathrm{t}}$ and $C r_{\mathrm{o}}$ are relative occupancies. In total 20 parameters were refined.

\begin{tabular}{|c|c|c|}
\hline \multirow[t]{2}{*}{ Parameter } & \multicolumn{2}{|c|}{ Sample } \\
\hline & S1 sample & S2 sample \\
\hline$a(\AA)$ & $8.333(3)$ & $8.351(1)$ \\
\hline$u(32 \mathrm{e})$ & $0.26173(11)$ & $0.2567(1)$ \\
\hline $\mathrm{Co}_{\mathrm{t}}(8 \mathrm{a})$ & $0.99(1)$ & $0.95(1)$ \\
\hline$C r_{\mathrm{o}}(16 \mathrm{~d})$ & $1.99(1)$ & $1.90(1)$ \\
\hline $\operatorname{BOV}\left(\AA^{2}\right)$ & $2.1(1)$ & $3.68(5)$ \\
\hline Profile function & \multicolumn{2}{|c|}{ Thompson-Cox-Hastings pseudo-Voigt } \\
\hline$Y\left(0.01^{\circ}\right)$ & $0.26(1)$ & $2.74(2)$ \\
\hline $0\left(0.01^{\circ}\right)$ & $0.12(3)$ & $0.2(1)$ \\
\hline Background function & \multicolumn{2}{|c|}{ Chebyshev polynomial (13 parameters) } \\
\hline$R_{f}(\%)$ & 1.34 & 2.62 \\
\hline$R_{B}(\%)$ & 1.62 & 2.68 \\
\hline$R_{w p}(\%)$ & 4.28 & 3.32 \\
\hline$R_{\exp }(\%)$ & 4.23 & 2.97 \\
\hline$\chi^{2}$ & 1.1 & 1.3 \\
\hline
\end{tabular}

between amorphous and nanocrystalline state are used for our investigation of the particle size dependence of magnetic phase transitions.

Temperature-dependent dc magnetization measurements reveal three magnetic phase transitions for sample S1 with a particle size of 26.9(1) $\mathrm{nm}$ [Fig. 2(a)]. Transition from the paramagnetic to the superparamagnetic (SPM) state occurs
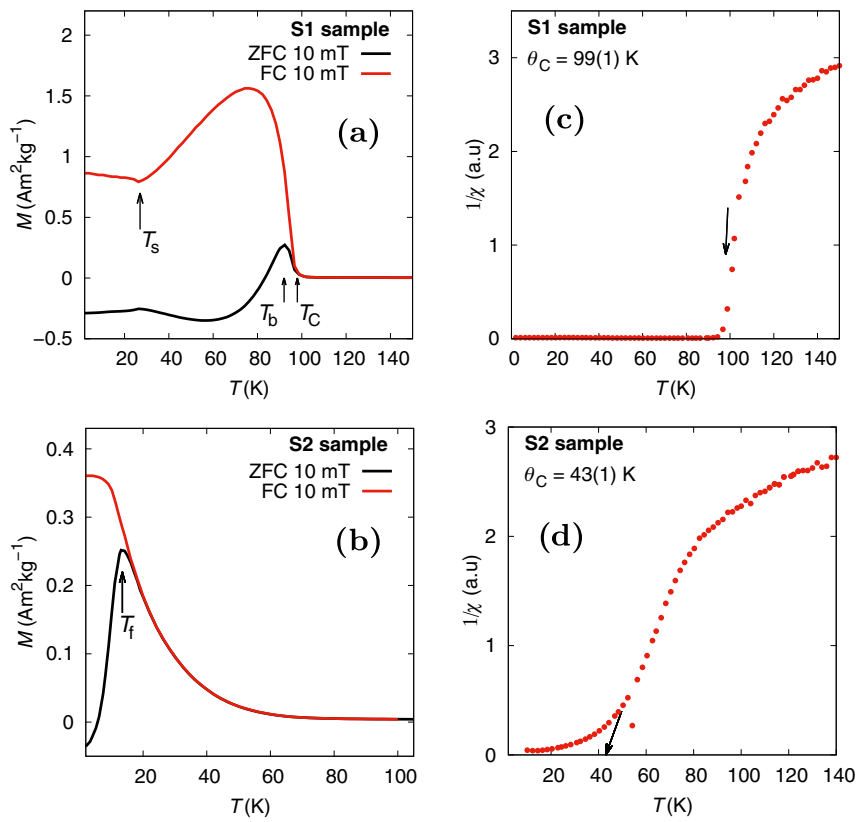

FIG. 2. (a) and (b) Temperature-dependent magnetization and (c) and (d) inverse susceptibility of the S1 and S2 samples. The asymptotic paramagnetic temperatures $\theta_{\mathrm{C}}$ of $99(1)$ and $43(1) \mathrm{K}$, respectively, were obtained from linear extrapolation of $T_{\max }(1 / \chi \rightarrow$ 0 ) in the paramagnetic regime (indicated by an arrow).
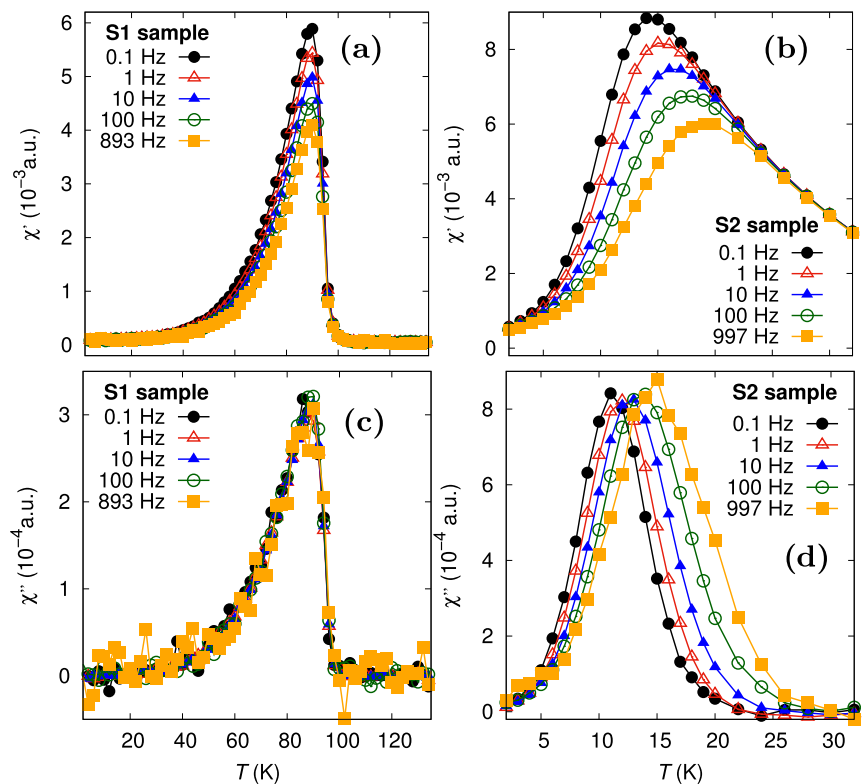

FIG. 3. Temperature dependence of (a) and (b) real and (c) and (d) imaginary part of ac susceptibility recorded at various frequencies of S1 and S2 samples.

at the asymptotic Curie temperature $\theta_{\mathrm{C}}=99(1) \mathrm{K}$ obtained from extrapolation of the inverse magnetic susceptibility $\chi^{-1}$ [Fig. 2(c)]. This magnetic phase transition is reflected in isothermal magnetization measurements [Supplemental Material (SM) Fig. 1(a) [30]] by a transition of the linear, paramagnetic behavior to a Langevin-like behavior. A Langevin fit of the superparamagnetic (SPM) state at $95 \mathrm{~K}$ (corresponding to a reduced temperature of $T_{r}=T / T_{C}=0.98$ ) reveals a particle moment of $\mu=946(2) \mu_{B}$ and a spontaneous magnetization of $M_{s}=10.4(3) \mathrm{kA} / \mathrm{m}$. This is in reasonable agreement with the bulk magnetization of $38.4 \mathrm{kA} / \mathrm{m}$ at 27 $\mathrm{K}\left(T_{r}=0.28\right)$, considering the rapid decrease of the order parameter towards the Curie temperature (97 K [29]). Below the blocking temperature $T_{\mathrm{b}}=92 \mathrm{~K}$, the particles are superparamagnetically blocked with ferrimagnetic intraparticle long-range order. This is associated with moderate hysteresis observed in isothermal magnetization measurements with a coercive field of $\mu_{0} H_{\mathrm{C}}=0.2 \mathrm{~T}$ at $2 \mathrm{~K}$ [30]. The excess susceptibility in even a large applied magnetic field is attributed to spin disorder, likely at the NP surface [31]. Transition to the noncollinear spin structure is observed at $T_{\mathrm{s}}=27$ $\mathrm{K}$, in excellent agreement with the bulk material [9-11]. It is accompanied by increased coercivity $\left(\mu_{0} H_{\mathrm{C}}=0.8 \mathrm{~T}\right.$ at $2 \mathrm{~K}$ ) in isothermal magnetization measurements [SM Fig. 1(b) [30]]. In contrast to previous studies of polycrystalline cobalt chromite samples, the so-called lock-in transition, a magnetic phase transition to the ground state of magnetic order [11] is not observed in our nanoparticle sample. The negative ZFC magnetization at low temperature is attributed to a difference in the temperature dependence of the A and B sublattices, causing a change of sign of the resulting net magnetization at the so-called compensation temperature [32].

Ac susceptometry measurements [Figs. 3(a) and 3(c)] reveal a sharp peak at transition $T_{\mathrm{b}}=92 \mathrm{~K}$ (blocked state). The blocking temperature is frequency independent in both real 

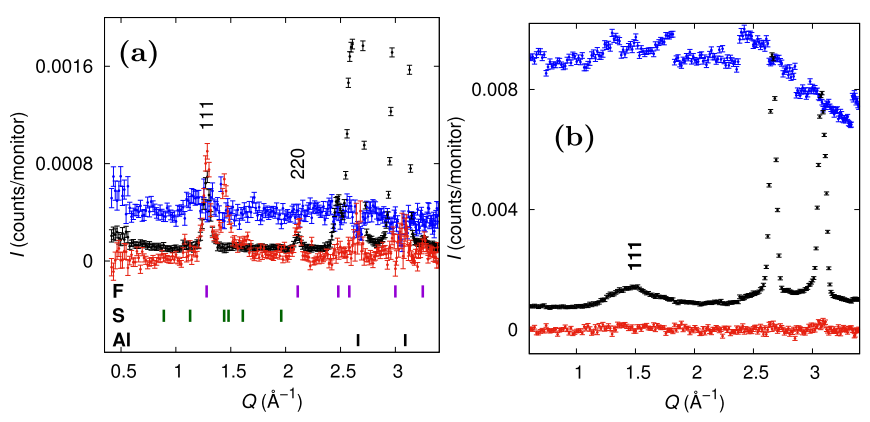

FIG. 4. Polarized neutron scattering with XYZ polarization analysis recorded at $5 \mathrm{~K}$ for a) S1 sample and b) S2 sample. Separated nuclear coherent (black dots), pure magnetic (red dots) and spin incoherent scattering contribution (blue dots) are shown. The position of fundamental (violet), satellites (green) and aluminum (black) reflections are indexed with vertical lines.

and imaginary parts of the ac susceptibility, confirming the absence of significant interparticle interactions in sample S1.

In order to elucidate the microscopic origin of the observed magnetic phase transitions of the $S 1$ sample, polarized neutron scattering with XYZ polarization analysis was carried out. Figure 4(a) presents the separated scattering contributions obtained for $\mathrm{S} 1$ at a base temperature of $5 \mathrm{~K}$. Data are presented in absolute scale in order to highlight the relative amount of the different scattering contributions. In S1 the magnetic scattering cross section is comparable in intensity with the nuclear coherent scattering cross section, accompanied by a low amount of spin-incoherent scattering. The spin-incoherent scattering cross section is nearly constant, confirming a successful separation and reference calibration. The Bragg reflections observed in the nuclear coherent scattering cross section are indexed according to the $\mathrm{CoCr}_{2} \mathrm{O}_{4}$ spinel structure with a lattice parameter of $a=8.32(1) \AA$, in reasonable agreement with the PXRD results. Additional Bragg reflections observed at $Q=2.7$ and $3.1 \AA^{-1}$ correspond to the aluminum sample holder. In the magnetic scattering cross section, the $\left(\begin{array}{lll}1 & 1 & 1\end{array}\right)$ and ( $\left.\begin{array}{lll}2 & 2 & 0\end{array}\right)$ fundamental reflections are observed at the same position as in the nuclear coherent scattering contribution with the same width as nuclear coherent reflections $(0.021(5)$ $\AA^{-1}$ ) and comparable scattering intensity. In addition, satellite reflections attributed to the spiral magnetic structure can be observed at $1.41,1.61$, and $1.93 \AA^{-1}$. The region from 2.6 to $3.1 \AA^{-1}$ was excluded from fit due to residual nuclear coherent reflection of the aluminum container, which cannot entirely be separated by polarization corrections.

The temperature-dependent differential magnetic scattering cross sections of S1 are presented in Fig. 5(a) for the temperature range from 5 to $95 \mathrm{~K}$. Magnetic fundamental reflections are detected up to $97 \mathrm{~K}$ in agreement with the Curie transition observed macroscopically. This corresponds to the collinear magnetic order of the ferrimagnetic spinel structure. The integral intensity of the magnetic fundamental reflections diminishes and broadens towards Curie temperature of $97 \mathrm{~K}$ [Fig. 5(d)]. Magnetic satellites are observed up to a temperature of $25 \mathrm{~K}$ in agreement with the macroscopically observed spin spiral transition at $T_{\mathrm{S}}=27 \mathrm{~K}$ which is comparable to the transition temperature of the conical spiral phase found for
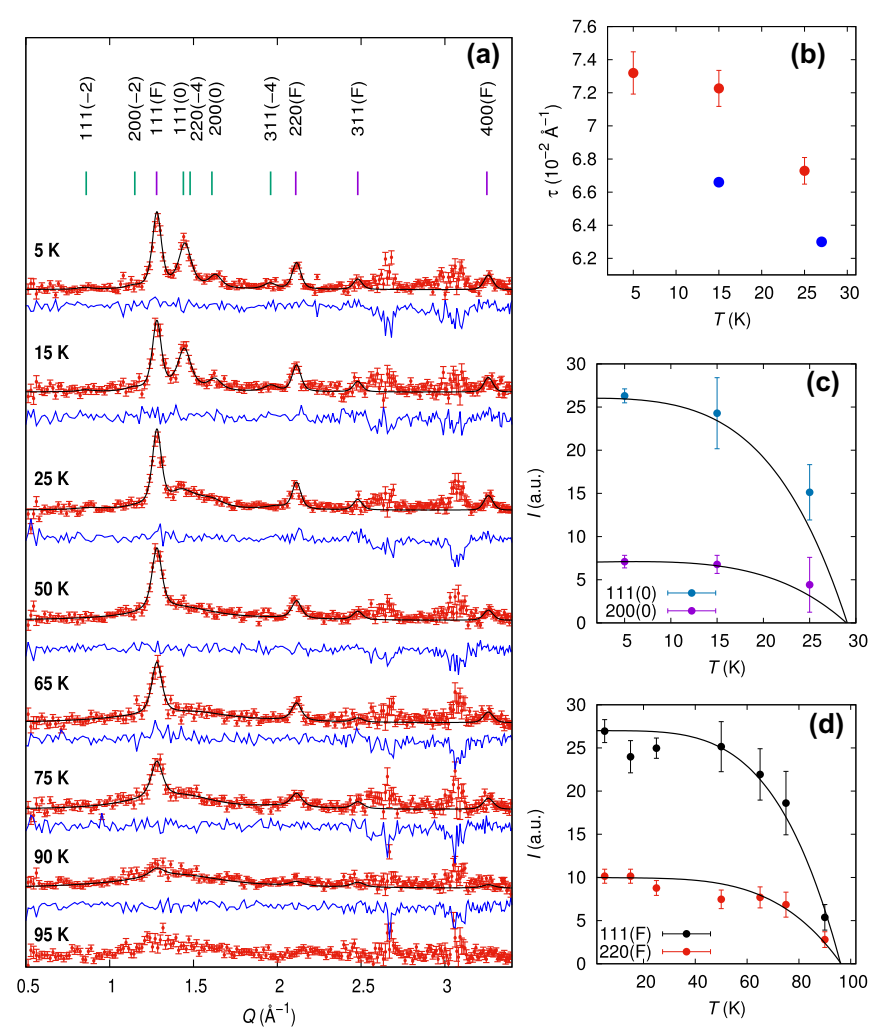

FIG. 5. (a) Magnetic scattering of S1 over a temperature range of 5-95 K. Red points represent the measured data, black line is LeBail fit, blue line shows the residual, and green and violet vertical lines correspond to the positions of magnetic satellites and fundamental reflections, respectively. (b) Temperature dependence of the propagation vector $\tau$ (red and blue circles correspond to propagation vector for nanocrystalline (sample S1) and single crystal cobalt chromite [11], respectively). (c) Temperature dependence of 111(0) and 200(0) magnetic satellites with the black line as a guide to the eye. (d) Temperature dependence of 111(F) and 220(F) fundamental reflections from collinear magnetic structure. The black line represents a guide to the eye.

bulk material $[11,33]$. The satellite reflections are broader than the nuclear coherent and fundamental magnetic reflections, suggesting short-range order of the spiral component in agreement with results found by Nair et al. [34]. In order to extract the spin spiral propagation vector, the magnetic fundamental and satellite reflection positions were determined using a Le Bail fit according to the LKDM and Hastings and Corliss models that have been applied to the bulk material $[35,36]$. In the structure factor for a conical spin structure, magnetic satellite scattering occurs at $\vec{K} \pm \vec{\tau}$ and $\vec{K}$ corresponding to the momentum transfer of the fundamental reflections. The model provides a simple scheme for indexing satellite reflections, which have nonintegral Miller indices expressed as $h k l(N)$ with $N=h u+k v+l w$. The lattice plane distance of the magnetic satellite reflections is given by

$1 / d_{H}^{2}=(\vec{K} \pm \vec{\tau})^{2}=1 / d_{H}^{2}+\tau^{2} \pm\left(\sqrt{2}|\vec{\tau}| / a_{0}\right)(h u+k v+l w)$,

where $h, k$, and $l$ are the Miller indices of $\vec{K}, \vec{\tau}$ is the propagation vector with its components $u, v$, and $w, 1 / d_{H}$ and $1 / d_{H}^{\prime}$ correspond to the lattice plane distance of the 
nuclear and magnetic reflection, respectively, and $a_{0}$ is the unit cell edge. The $\left[\begin{array}{lll}1 & 1 & 0\end{array}\right]$ direction of the propagation vector has been chosen as this direction was observed in bulk [33]. The incommensurate propagation vector of the spiral component was determined as $(\tau \tau 0)$ with $\tau=0.073(1)$ $\AA^{-1}$ at $5 \mathrm{~K}$ and a structural coherence length of the spiral order of $\pi / \sqrt{2 \tau^{2}}=3.0(1) \mathrm{nm}$. The propagation vector $\tau$ is strongly temperature dependent near the transition temperature $T_{\mathrm{s}}$ [Fig. 5(b)]. However, in contrast to the bulk material where $\tau$ locks in at $\tau=2 / 3\left(T_{\text {lock-in }}=14.5 \mathrm{~K}\right)$, the propagation vector is significantly larger and incommensurate in the entire spin spiral phase of the nanosized sample. The integral intensity of the (200(0), 111(0)) magnetic satellite reflections [Fig. 5(c)] decreases significantly towards the magnetic phase transition at $T_{\mathrm{s}}=27 \mathrm{~K}$ as expected for the magnetic order parameter. Above $T_{\mathrm{s}}$, an enhanced diffuse scattering contribution is still observed [Fig. 5(a)] that is attributed to magnetic spin fluctuations.

The sample S2 with smaller particle size of 3.1(2) nm and coherent crystallite size of $2.9(2) \mathrm{nm}(a=8.358(1) \AA)$ reveals strikingly different magnetic properties in comparison to S1. Below $18 \mathrm{~K}$ [Fig. 2(b)], the ZFC/FC magnetization measurements conform to a spin glass without long-range order with a freezing temperature of $T_{\mathrm{f}}=15 \mathrm{~K}$ (maximum of ZFC) and asymptotic paramagnetic temperature of $\theta_{\mathrm{C}}=43(1)$ $\mathrm{K}$ [Fig. 2(d)]. In the spin glass state below $T_{\mathrm{f}}$, hysteresis appears with a large coercive field of $0.8 \mathrm{~T}$ at $2 \mathrm{~K}$ [SM Fig. 1(b) [30]]. The broad hysteresis is attributed to strong interparticle interaction. This is supported by magnetization measurements of the same sample diluted in toluene, i.e., excluding interparticle interactions. No hysteresis is observed in the dispersion even at $5 \mathrm{~K}$ [SM Fig. 2(a) [30]], and temperature dependence of magnetization [SM Fig. 2(b) [30]] shows lowering of both the freezing temperature $T_{\mathrm{f}}=9(1) \mathrm{K}$ and the asymptotic paramagnetic temperature $\theta_{\mathrm{C}}=19(6) \mathrm{K}$ in comparison with powder measurements. The real and imaginary parts of ac susceptibility measurements of the powder sample [Figs. 3(b) and 3(d)] reveal a strong frequency dependence of the transition temperature. According to Binder [37], this behavior generally indicates an atomic spin glass with relaxation time in the range of $t \sim 10^{-12}-10^{-14} \mathrm{~s}$. Superparamagnetic and weakly interacting NPs known as super spin glass reveal a slower relaxation time of $t \sim 10^{-7}-10^{-10} \mathrm{~s}$ in comparison with the atomic spin glass, which was found for weakly interacting $\mathrm{CoCr}_{2} \mathrm{O}_{4}$ NPs [38]. Even slower relaxation times of $t \sim 10^{-5}$ $10^{-6} \mathrm{~s}$ are attributed to a cluster glass behavior resulting from strong interparticle interactions as established for maghemite $\left(\gamma-\mathrm{Fe}_{2} \mathrm{O}_{3}\right)$ NPs [39]. Applying the Vogel Fulcher law [40] a relaxation time of $t=2.8 \times 10^{-5} \mathrm{~s}$ is obtained, indicating a collective cluster glass behavior for our S2 sample.

In order to prove the suggested cluster glass behavior, polarized neutron diffraction with XYZ polarization analysis was carried out. The diffractogram at $5 \mathrm{~K}$ [Fig. 4(b)] reveals a very broad fundamental $\left(\begin{array}{lll}1 & 1 & 1\end{array}\right)$ reflection in the nuclear coherent scattering contribution, corresponding to a small structurally coherent grain size of 2.9(2) $\mathrm{nm}$ and a lattice parameter of 8.35(2) $\AA$, in excellent agreement with the results obtained from PXRD. In contrast to the S1 sample, here the incoherent scattering contribution is significantly higher, due to a larger amount of organic ligand material. No magnetic scattering contributions, neither fundamental nor satellite reflections, are observed within the experimental uncertainties at the base temperature. This information is only accessible with XYZ polarization analysis, which allows us to efficiently separate nuclear coherent, spin-incoherent, and magnetic scattering contributions [41]. XYZ polarization analysis can be applied in order to remove a strong incoherent scattering background from a small coherent or magnetic scattering signal [42-44]. Similar behavior for spin glasses with no magnetic scattering was found also for spinel $\mathrm{Co}_{2} \mathrm{RuO}_{4}$ [45] and more recently for $\mathrm{La}_{3} \mathrm{Ni}_{2} \mathrm{NbO}_{9}$ and $\mathrm{La}_{3} \mathrm{Ni}_{2} \mathrm{TaO}_{9}$ compounds [46]. For spinel $\mathrm{Co}_{2} \mathrm{RuO}_{4}$ [45], the spin-glass behavior was attributed to a very weak interaction of the ions in octahedral sites and strong disorder of their occupancy. Structural disorder might also be one of the reasons for the magnetic spin glass behavior observed for the S2 sample. The larger lattice parameter compared to the $\mathrm{S} 1$ sample and the bulk material indicates strong structural disorder that is likely associated with a certain degree of spinel inversion. Interparticle correlations as present in a magnetic cluster glass, with magnetic short-range order in the range of few nanometers, are expected to generate diffuse magnetic scattering in the small-angle range that is not accessible with our diffraction experiments. Both samples S1 and $\mathrm{S} 2$ have a comparable saturation magnetization [30], such that magnetic scattering arising from a coherently magnetized volume should have comparable magnitude as the observed nuclear coherent scattering. Hence, no Bragg scattering is observed, as there are no correlations at the atomic scale in the magnetic cluster glass. The absence of any fundamental magnetic reflections is in agreement with the existence of a cluster glass magnetic phase (i.e., mictomagnet), where the long-range ferromagnetic order is incipient at low temperature.

\section{CONCLUSION}

By means of polarized neutron scattering and magnetization measurements, the magnetic phase transitions in nanocrystalline $\mathrm{CoCr}_{2} \mathrm{O}_{4}$ of two different particle sizes in the intermediate regime between polycrystalline and amorphous state are investigated. For the $\mathrm{S} 1$ sample with a mean particle diameter of 26.9(1) nm, magnetic phase transitions towards collinear and spin spiral magnetic states are observed. The found Curie and spin spiral transition temperatures are in excellent agreement with the bulk material. However, no evidence for the lock-in transition is found, and the spin spiral propagation vector $\tau$ remains significantly larger than in bulk material and incommensurate throughout the entire phase range. The incommensurability of the propagation vector is likely due to varying magnitudes of the average magnetic moments and the cone angles for each sublattice with temperature. In order to resolve the origin of this incommensurability in more detail, further experimental data on a series of grain sizes are required. In contrast, neither ferrimagnetic order nor noncollinear magnetic order exists in the $\mathrm{S} 2$ sample with smaller $\mathrm{CoCr}_{2} \mathrm{O}_{4} \mathrm{NPs}$, where cluster glass behavior is found. The absence of any fundamental magnetic reflections at base temperature indicates that spins are frozen randomly. Local structural disorder is presumably the main source for this effect. The noncollinear spin spiral phase cannot exist without collinear magnetic order in the NPs and is consequently not found here. In order to verify the impact of the particle size on the magnetic phase transitions 
more closely, the challenge of synthesizing $\mathrm{CoCr}_{2} \mathrm{O}_{4}$ NPs with a series of grain sizes and good crystallinity needs to be met. In this way, a potential correlation between the particle diameter and the structural coherence length of the spin spiral structure may be revealed, thereby paving the way towards understanding the critical size limit for the onset of ferroelectricity and thus multiferroic effects in nanosized $\mathrm{CoCr}_{2} \mathrm{O}_{4}$.

\section{ACKNOWLEDGMENTS}

This work is based upon experiments performed at the DNS instrument operated by Jülich Centre for Neutron Science (JCNS) at the Heinz Maier-Leibnitz Zentrum (MLZ),
Garching, Germany. We thank Jana Kalbáčová Vejpravová for the ac susceptibility measurements, which were performed in MLTL (see htpp://mltl.eu), supported within the program of Czech Research Infrastructures (project no. LM2011025). We acknowledge M. Herlitschke for performing CCMS measurements at the Forschungszentrum Jülich. P. Fitl and J. Vlček thank the Czech Science Foundation project no. 18-09347S and support by Ministry of Education, Youth and Sports of the Czech Republic in the frame of project LTC17058 and by COST Action CA15107 MultiComp. Financial support from the Deutsche Forschungsgemeinschaft (DFG: Emmy Noether Grant DI1788/2-1) as well as the UoC Key Profile Arca III Quantum Matter \& Materials is gratefully acknowledged.
[1] M. Mostovoy, Phys. Rev. Lett. 96, 067601 (2006).

[2] M. Fiebig, J. Phys. D 38, R123 (2005).

[3] T. Kimura, S. Ishihara, H. Shintani, T. Arima, K. T. Takahashi, K. Ishizaka, and Y. Tokura, Phys. Rev. B 68, 060403 (2003).

[4] T. Goto, T. Kimura, G. Lawes, A. P. Ramirez, and Y. Tokura, Phys. Rev. Lett. 92, 257201 (2004).

[5] D. Higashiyama, S. Miyasaka, N. Kida, T. Arima, and Y. Tokura, Phys. Rev. B 70, 174405 (2004).

[6] N. Hur, S. Park, P. A. Sharma, J. S. Ahn, S. Guha, and S. W. Cheong, Nature (London) 429, 392 (2004).

[7] E. Bousquet and A. Cano, J. Phys.: Condens. Matter 28, 123001 (2016).

[8] S. W. Cheong and M. Mostovoy, Nat Mater. 6, 13 (2007).

[9] I. Kim, Y. S. Oh, Y. Liu, S. H. Chun, J.-S. Lee, K.-T. Ko, J.-H. Park, J.-H. Chung, and K. H. Kim, Appl. Phys. Lett. 94, 042505 (2009).

[10] L. J. Chang, D. J. Huang, W.-H. Li, S.-W. Cheong, W. Ratcliff, and J. W. Lynn, J. Phys.: Condens. Matter 21, 456008 (2009).

[11] Y. Yamasaki, S. Miyasaka, Y. Kaneko, J.-P. He, T. Arima, and Y. Tokura, Phys. Rev. Lett. 96, 207204 (2006).

[12] T. Kimura, T. Goto, H. Shintani, K. Ishizaka, T. Arima, and Y. Tokura, Nature (London) 426, 55 (2003).

[13] G. Lawes, B. Melot, K. Page, C. Ederer, M. A. Hayward, T. Proffen, and R. Seshadri, Phys. Rev. B 74, 024413 (2006).

[14] Y. J. Choi, J. Okamoto, D. J. Huang, K. S. Chao, H. J. Lin, C. T. Chen, M. van Veenendaal, T. A. Kaplan, and S.-W. Cheong, Phys. Rev. Lett. 102, 067601 (2009).

[15] L. Kumar, P. Mohanty, T. Shripathi, and C. Rath, Nanosci. Nanotechnol. Lett. 1, 199 (2009).

[16] C. Rath and P. Mohanty, J. Supercond. Novel Magn. 24, 629 (2011).

[17] D. Gingasu, I. Mindru, D. C. Culita, L. Patron, J. M. CalderonMoreno, P. Osiceanu, S. Preda, O. Oprea, V. Parvulescu, V. Teodorescu et al., Mater. Res. Bull. 62, 52 (2015).

[18] D. Zákutná, A. Repko, I. Matulková, D. Nižňanský, A. Ardu, C. Cannas, A. Mantliková, and J. Vejpravová, J. Nanopart. Res. 16, 2251 (2014).

[19] D. Kumar, J. K. Galivarapu, A. Banerjee, K. S. Nemkovski, Y. Su, and C. Rath, Nanotechnology 27, 175702 (2016).

[20] J. K. Galivarapu, D. Kumar, A. Banerjee, V. Sathe, G. Aquilanti, and C. Rath, RSC Adv. 6, 63809 (2016).

[21] J. K. Galivarapu, D. Kumar, A. Banerjee, and C. Rath, IEEE Trans. Magn. 52, 6000506 (2016).
[22] H. Cui, M. Zayat, and D. Levy, J. Sol-Gel Sci. Technol. 35, 175 (2005).

[23] J. Rodriguez-Carvajal, Physica B 192, 55 (1993).

[24] W. Schweika and P. Böni, Physica B 297, 155 (2001).

[25] Y. Su, K. Nemkovskiy, and S. Demirdis, J. Large-Scale Res. Facil. 1, A27 (2015).

[26] O. Schärpf and H. Capellmann, Phys. Status Solidi A 135, 359 (1993).

[27] A. Glavic, Plot.py—data plotting and evaluation software, URL https://www.sourceforge.net/projects/plotpy/.

[28] P. Thompson, D. E. Cox, and J. B. Hastings, J. Appl. Crystallogr. 20, 79 (1987).

[29] N. Menyuk, K. Dwight, and A. Wold, J. Phys. France 25, 528 (1964).

[30] See Supplemental Material at http://link.aps.org/supplemental/ 10.1103/PhysRevB.98.064407 for the isothermal magnetization of samples S1 and S2 at various temperatures and the magnetic field and temperature-dependent magnetization of a dilute toluene dispersion of S2 NP.

[31] R. H. Kodama, A. E. Berkowitz, E. J. McNiff, and S. Foner, J. Appl. Phys. 81, 5552 (1997).

[32] L. Néel, Ann. Phys. 12, 139 (1948).

[33] K. Tomiyasu, J. Fukunaga, and H. Suzuki, Phys. Rev. B 70, 214434 (2004).

[34] H. S. Nair, Z. Fu, J. Voigt, Y. Su, and T. Brückel, Phys. Rev. B 89, 174431 (2014).

[35] D. H. Lyons, T. A. Kaplan, K. Dwight, and N. Menyuk, Phys. Rev. 126, 540 (1962).

[36] J. M. Hastings and L. M. Corliss, Phys. Rev. 126, 556 (1962).

[37] K. Binder and A. P. Young, Rev. Mod. Phys. 58, 801 (1986).

[38] Z. Tian, C. Zhu, J. Wang, Z. Xia, Y. Liu, and S. Yuan, J. Magn. Magn. Mater. 377, 176 (2015).

[39] D. Fiorani, J. Dormann, R. Cherkaoui, E. Tronc, F. Lucari, F. D’Orazio, L. Spinu, M. Nogues, A. Garcia, and A. Testa, J. Magn. Magn. Mater. 196-197, 143 (1999).

[40] S. Shtrikman and E. P. Wohlfarth, Phys. Lett. A 85, 467 (1981).

[41] J. R. Stewart, P. P. Deen, K. H. Andersen, H. Schober, J. Barthélémy, J. M. Hillier, A. P. Murani, T. Hayes, and B. Lindenau, J. Appl. Crystallogr. 42, 69 (2009).

[42] S. Disch, R. P. Hermann, E. Wetterskog, A. A. Podlesnyak, K. An, T. Hyeon, G. Salazar-Alvarez, L. Bergström, and T. Brückel, Phys. Rev. B 89, 064402 (2014). 
[43] G. Goracci, A. Arbe, A. Alegría, W. Lohstroh, Y. Su, and J. Colmenero, J. Chem. Phys. 143, 094505 (2015).

[44] C. Gerstl, M. Brodeck, G. J. Schneider, Y. Su, J. Allgaier, A. Arbe, J. Colmenero, and D. Richter, Macromolecules 45, 7293 (2012).
[45] D. Mandrus, V. Keppens, and B. Chakoumakos, Mater. Res. Bull. 34, 1013 (1999).

[46] C.-M. Chin, P. D. Battle, S. J. Blundell, E. Hunter, F. Lang, M. Hendrickx, R. P. Sena, and J. Hadermann, J. Solid State Chem. 258, 825 (2018). 